\title{
Mediated generalization and interference across five grade levels'
}

J. KENT DAVIS

UNIVERSITY OF WISCONSIN

The present study employed a mixed-list mediation paradigm in which all Ss were administered mediation, interference and control pairs. Ten Ss from each of 5 grade levels $(2,4$, 6,8 , and college) followed a paired-associate anticipation method in learning a list of 9 high frequency word pairs. Analysis of errors failed to demonstrate any presence of an interaction between grade level and conditions. The third stage leaming results showed that all grade levels performed best on the mediation pairs, intermediate on the control pairs, and poorest on the interference pairs, with the exception of of a slight reversal between control and mediation pairs for eighth graders.

Subjects of various grade levels have neen employed in mediation studies (Norcross \& Spiker, 1958; Berkson \& Cantor, 1960; Wismer \& Lipsitt, 1964; and Wu \& Lipsitt, 1956). Most of these studies, however, have been concerned primarily with the phenomena of mediation and have not independently considered the grade level of the Ss. Examined here is the effect of mediation, interference and control conditions in paired-associate learning by five grade levels of Ss.

Method

A total of $50 \mathrm{Ss}$ participated in the experiment; $10 \mathrm{Ss}$ each from grades $2,4,6$, and 8 were obtained from a local grade and junior high school, and 10 college Ss were obtained from an introductory educational psychology class.

The stimulus materials consisted of three-letter, high frequency nouns (rated AA on the Thorndike-Lorge word count). From a total of 30 words meeting this criterion, 10 lists were constructed. Word pairings were randomly determined with the restriction that logical connections were to be avoided as much as possible. Each of the 10 lists contained nine word pairs; three pairs followed the $A-B, B-C, A-C$ mediation paradigm; three pairs followed the $\mathrm{A}-\mathrm{B}, \mathrm{B}-\mathrm{C}, \mathrm{A}-\mathrm{Cr}$ interference paradigm; and three pairs followed the A-B, D-C, A-C control paradigm. For each of the three stages in each of the 10 lists, four random orders were constructed in order to minimize serial learning. The stimulus words and the stimulus-response word pairs were typed on a mimeograph stencil and then mounted in a $2 \times 2$ in. slide mount.

Each $\mathrm{S}$ was tested individually and run to a criterion of two successive correct runs through the list. Nine paired associates were presented following the anticipation method via a Kodak Carousel slide projector at a 8:8 sec. rate with no intertrial interval. The interstage interval was approximately $1 \mathrm{~min}$. in duration.
The design of the study consisted of a 3 by 5 by 10 incomplete factorial design with three types of word pairs (mediation, interference, and control conditions), five grade levels $(2,4,6,8$, and college), and 10 randomly constructed lists of nine items each. One $S$ from each of the five grade levels was run under one of the 10 randomly constructed lists.

\section{Resulis and Discussion}

An analysis of variance was performed on the number of errors to criterion for each of the three stages of learning. The over-all mean number of errors for the mediation, interference, and control pairs during each stage of learning is presented in Fig. 1.

\section{Stage 1 Learning}

During stage I learning, the over-all effect of type of word pair was not significant $(F<1)$, indicating that type of word pairs were of comparable difficulty during initial learning. The over-all effect of grade level was significant $(F=2.776, d f=4 / 36, p<.05)$. The grade level by type of word pair interaction was not significant. Stage II Learning

As can be seen in Fig. 1, type of word pair was a significant source of variance for stage II learning $(F=15.91, d f=2 / 18, p<.01)$. Subsequent analysis by means of the Newman-Keuls procedure revealed that the control pairs (D-C) were significantly easier to learn than the mediation and interference pairs $(B-C)$ which did not differ from each other. The most plausible explanation for this finding is that the learning of the $B-C$ pairs requires unlearning of the backward association $\mathrm{B}-\mathrm{A}$, while the learning of the $\mathrm{D}-\mathrm{C}$ pairs does not

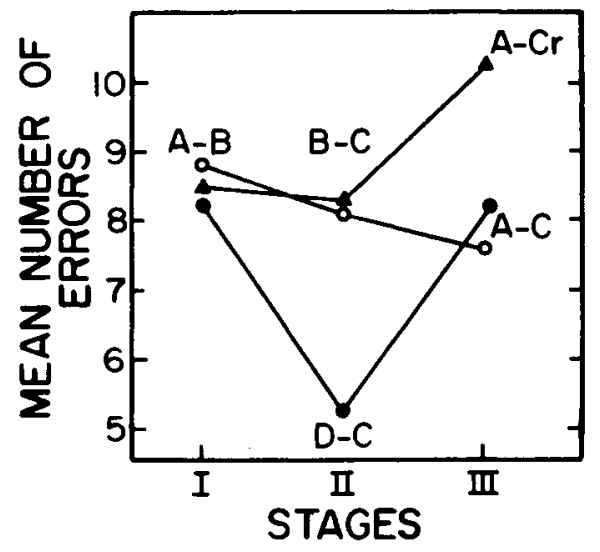

Fig.. 1. Mean number of errors in the three learning stages. 


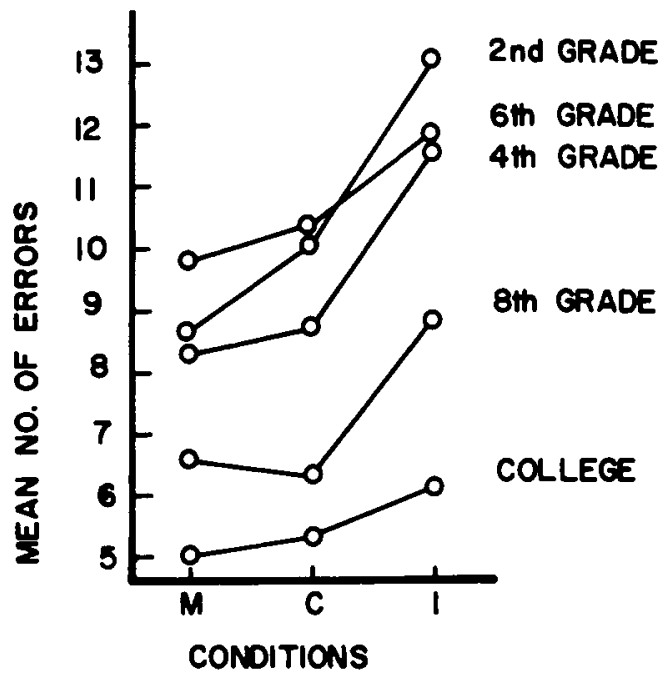

Fig. 2. Mean number of errors in stage III learning for mediation (M), interference (I), and control (C) conditions for each grade level.

(Postman, 1962). The grade level effect was again significant $(F=3.367, \mathrm{df}=4 / 36, \mathrm{p}<.05)$, but because the grade level by type of word pair interaction was not significant, only the main effect of type of word pairs is shown in Fig. 1.

\section{Stage III Learning}

The type of word pair effect was also significant $(\mathrm{F}=$ 15.81, df $=2 / 18, p<.01$ ) for stage III learning (see Fig. 1). The interference condition (A-Cr) resulted in significantly poorer performance than did the mediation and control conditions as indicated by the Newman-Keuls procedure. Although the difference between the mediation and control condition is in the direction predicted by the mediation hypothesis, it is not statistically reliable. When the mean percent correct responses was examined across blocks of three trials, however, the mediation condition (mean of $64.4 \%$ correct) was significantly $(t=1.805, p<.05)$ different from the control condition (mean of $56.0 \%$ correct) over the first block of three trials, indicating that facilitation as a result of the mediation paradigm was present butonly early in stage III learning. As in stage I and II learning, grade level was significant $(F=3.24, d f=4 / 36, p<.05)$, while the interaction of grade level by type of word pair was not.

Figure 2 presents the mean number of errors during stage III learning for each type of word pair for each grade level. The mediation hypothesis would predict that during stage III learning the over-all performance on the mediation pairs would be better than that for the control pairs, both of which would be better than that for the interference pairs. As can be seen in Fig. 2, all grades show the relationship predicted by the mediation hypothesis, with the exception of a reversal between the control and mediation conditions for eighth graders. Except for the eighth graders, each grade level performed better on the mediation pairs, intermediate on the control pairs and poorest on the interference pairs. Page's (1963) test $(L=69, p<.01)$ confirmed the predicted ordered relationship in terms of ease of learning.

If total errors are considered on stage III learning (collapsing across conditions), then there is a tendency for the sixth graders to perform the poorest. The trend showing sixth graders' performance as being inferior to that of the other grade levels might possibly be explained in terms of different associative strategies utilized (Martin, Boersma, \& Cox, 1965). It is possible that sixth grade represents a transition between those strategies which are the most efficient (used by eighth grade and college Ss) and those which are less efficient (used by second and fourth grade Ss). Thus the poorer performance of sixth graders would represent the utilization of a more efficient strategy but with less proficiency in the use of this strategy.

In summary, there were no differences between the three types of word pairs during stage I learning. During stage II learning, however, performance on the mediation and interference pairs was inferior to performance on the control pairs, but by stage III learning the mediation condition resulted in fewer errors than did the control and interference conditions. The absence of a significant grade level by type of word pair interaction suggests that there is no differential effect of the three types of word pairs upon Ss from different grade levels.

\section{References}

Berkson, G., \& Cantor, G. N. A study of mediation in mentally retarded and normal school children. J. educ. Psychol., 1960. $51,82-86$.

Martin, C. J., Boersma, F. J., \& Cox, D. L. A classification of associative strategies in paired-associate learning. Psychon. Sci., 1965, 3, 455-456.

Norcross, K. J., \& Spiker, C. C. Effects of mediated association on transfer in paired-associate learning. J. exp. Psychol., 1958, $55,129-134$.

Page, E. B. Ordered hypotheses for multiple treatments: a significance test for linear ranks. J. Amer. Statist.. Assoc., 1963, 58, 216-230.

Postman, L. Transfer of training as a function of experimental paradigm and degree of first-list learning. J. verbal Learn. verbal Behav., 1962, 1, 109-118.

Wismer, B., \& Lipsitt, L. Verbal mediation in paired-associate learning. J. exp. Psychol., 1964, 68, 441-448.

\section{Note}

1. This research was supported by the United States Office of Education, Department of Health, Education and Welfare, under the provisions of the cooperative research program center No. C-03/ contract OE 5-10-154 and CRP No. 2850. 